\title{
An Adaptable Prosthetic Wrist Reduces Subjective Workload
}

\author{
Authors: Nathaniel R. Olsen ${ }^{1}$, Jacob A. George ${ }^{1}$, Mark R. Brinton ${ }^{1}$, Michael D. Paskett ${ }^{1}$, David T. Kluger ${ }^{2}$, \\ Troy N. Tully ${ }^{3}$, Christopher C. Duncan ${ }^{4}$, Gregory A. Clark ${ }^{1}$ \\ 1. Department of Biomedical Engineering, University of Utah, Salt Lake City, UT \\ 2. Blackrock Microsystems, Salt Lake City, UT \\ 3. Department of Computer Science, Westminster College, Salt Lake City, UT \\ 4. Department of Physical Medicine and Rehabilitation, University of Utah, Salt Lake City, UT
}

\begin{abstract}
Many presently available prostheses lack a functional wrist. To fill this niche and to better understand the impact a wrist has in prosthetic functionality, we designed a low-cost, adaptable, 3Dprintable prosthetic wrist that can be adapted to various prosthetic hands and sockets. The wrist utilizes inexpensive but powerful servo motors to provide simultaneous and proportional control of two degrees of freedom: pronation/supination and flexion/extension or radial/ulnar deviation. Participants used both our wrist and a commercially available wrist (DEKA "LUKE" Arm) to complete a modified version of the clothespin relocation task with and without the wrists enabled. Through use of the NASA Task Load Index we found that both wrists significantly reduced the subjective workload associated with clothespin relocation task $(p<0.05)$. However, we found no significant difference in task completion speed, presumably due to compensation strategies. This inexpensive and adaptable prosthetic wrist can be used by amputees to reduce task workload, or by researchers to further explore the importance of wrist function.
\end{abstract}

\section{INTRODUCTION}

In 2005, there were an estimated 41,000 upper-limb amputees (excluding partial-hand) in the United States [1]. Amputation results in a life-long struggle with chronic pain, depression and functional disability [1-3]. The current standard of care, often a body-powered hook or myoelectric prosthetic hand, is unsatisfactory, causing up to $50 \%$ of amputees to abandon their prostheses [4]. Transradial amputees have expressed their top priorities for useful prostheses (in order of importance): wrist rotation, simultaneous movements, wrist deviation, wrist flexion/extension, increased automaticity [5]. Additional priorities include reduced weight, improved durability, and increased strength [5].

Functional wrist motion is important in reducing biomechanical strain. Without a wrist, amputees are forced to compensate with unnatural movements to complete standard activities of daily living (ADLs) [6-8]. The continual use of these motions causes further damage to the musculoskeletal system over long periods $[9,10]$. 
3D-printing has provided new and innovative solutions to address several of the above issues with prosthetics. For example, 3D-printing has considerably lowered the cost and weight of prostheses [11-13], and several 3D-printed prosthetic hands are currently available [14-16]. Traditionally, wrists have been excluded from the design of 3D-printed and commercial prosthetic arms to conserve space and to focus on the implementation of a more dexterous hand [17]. Commercially available prosthetic hands without an active wrist include the Michelangelo [18], i-limb Ultra [19], TASKA [20], and bebionic [21].

Although these commercially available prosthetic hands can be paired with a wrist, few commercial active wrists exist, and none provide more than 1 active degree of freedom [22,23]. There are many dedicated, passive wrists with varying degrees of freedom - such as the bebionic [21] - but these are unnatural since the wrist must be manually adjusted by the intact contralateral hand of the user - at which point, most unilateral tasks could have been completed more efficiently without involving the prosthetic.

To date, the only prosthetic hands fully integrated with an active wrist are the DEKA "LUKE" Arm [24] and the Modular Prosthetic Limb [25]. However, the DEKA "LUKE" Arm is expensive and the Modular Prosthetic Limb is not available to the public. To make it possible for more researchers to study prosthetic wrists, and for amputees to have access to an inexpensive alternative, there is a critical need for an adaptable, inexpensive prosthetic wrist.

Here we describe the development of an active, 3D-printed, inexpensive, and adaptable prosthetic wrist. We show that the implementation of this wrist as well as a commercial wrist

55 does not improve the time it takes to complete tasks but significantly reduces subjective workload in both cases by a factor of about 1.6. This work demonstrates the first standalone,

57 lightweight and 3D-printed prosthetic wrist capable of interfacing with various prosthetic hands 58 and sockets.

\section{METHODS}

\section{Design Criteria:}

61 Degrees of Freedom and Length: The human wrist moves in two degrees of freedom:

62 flexion/extension and radial/ulnar deviation. Pronation/supination is frequently attributed to the wrist; however, it is actually a function of the forearm. In prosthetic wrist design, 
pronation/supination is the most commonly included degree of freedom. Radial/ulnar deviation is included less frequently in wrist designs and is often viewed as the least important of the three degrees of freedom [8].

A transradial amputation occurs across the radial bone, below the elbow but above the wrist. Assuming the average transradial amputation occurs halfway down the forearm, it would be ideal for a wrist to not exceed half the length of the average human forearm. This length, averaged between males and females, is roughly $12.5 \mathrm{~cm} \mathrm{[26].} \mathrm{By} \mathrm{limiting} \mathrm{the} \mathrm{length} \mathrm{of} \mathrm{the} \mathrm{wrist}$ in this way, the wrist would be more easily integrated with a prosthetic hand without dramatic increases to the length of the prosthetic device.

Adaptable: Existing wrists are not easily adaptable to multiple hands. The Ottobock Quick Disconnect Wrist (Otto Bock HealthCare LP, Austin, TX) fits a variety of hands, but prostheses not equipped with the Ottobock Quick Disconnect Wrist cannot be used with this wrist. For a prosthetic wrist to fit with a wide variety of devices, the wrist needs to be adaptable on both the proximal and distal ends. The proximal end needs to be adaptable to differing sockets and the distal end to various prosthetic hands. This modularity can be easily facilitated with 3D-printing.

Lightweight: Active, 2-degree-of-freedom prosthetic wrists that can be added to hands weigh between 95 to 500 grams [23]. As prosthetic device weight is correlated with abandonment, an active wrist should be as light as possible, certainly not exceeding 500 grams [4,27].

Functional: The device would need to be strong enough to lift external objects and perform tasks. One study showed that the average maximum wrist flexion and extension torques at neutral position for the human arm was $8.0 \pm 3.0 \mathrm{~N}^{*} \mathrm{~m}$ and $4.6 \pm 1.0 \mathrm{~N} * \mathrm{~m}$, respectively [28]. Ideally, a prosthetic wrist, would match this level of functionality. However, commercially available options are limited by space constraints, availability, and cost. As such, the motors must be as strong but small and lightweight.

\section{Device Design:}

A prosthetic wrist was designed in Solidworks (Solidworks, Waltham, MA) (Figure 1A) and subsequently assembled (Figure 1B). The body of the wrist consists of an interlocking rotation mechanism and cap. The interlocking rotation mechanism was designed to be 3Dprinted with dissolvable support material, polyvinyl alcohol (PVA), between the interlocking 
parts. Once the support material is dissolved, the 2 subparts can freely rotate (Figure 1D). Each of these subparts houses a servo motor and the proximal subpart also houses an Adafruit Trinket M0 microcontroller (Adafruit Industries, New York, NY). The proximal servo motor is responsible for providing rotation between the 2 subparts. An Actobotics 525130 servo hub horn (RobotZone, Winfield, KS) is mounted to the proximal motor which is then fixed to the distal part via 4 screws. The distal servo motor is responsible for the additional degree of freedom (flexion/extension or radial/ulnar deviation) and has a servo hub horn mounted to it. The servo hub horn is fixed to the adaptable hand attachment portion of the wrist using 4 screws. Actobotics 545372 servo hub spacers (RobotZone, Winfield, KS) were used to provide proper spacing between the servo horn and the adaptable hand attachment portion of the wrist. The wrist can be manually adjusted to provide radial/ulnar deviation instead of flexion/extension. This is done by rotating the hand to the desired orientation before fixing it to the wrist. This modification changes the neural position of the hand 90 degrees. Because of this, reorienting the attachment of rotational servo motor the servo hub horn would be necessary.

Degrees of Freedom and Length: Because of the servo size and the length constraint of $12.5 \mathrm{~cm}$, we could implement only a 2-degree-of-freedom wrist. Pronation/supination was included in the design because it is the most frequently implemented degree of freedom. The wrist was designed to be in series with either flexion/extension or radial/ulnar deviation: either can be selected by attaching the prosthetic hand of choice in the desired orientation. The length of the device without any modifications to the proximal and distal ends is $11.8 \mathrm{~cm}$ (Table 1).

114 attachments. CAD design and 3D-printing can be used to allow for rapid adaptation to the distal and proximal ends of the device (Figure 1C and 3E). The distal hand adaptor was designed with

116 a flat surface so modified attachments can be easily printed to fit new hands to the wrist.

117 Likewise, the proximal end was designed to be easily modified to fit various sockets. inexpensive and lightweight—weighing only about 360 grams (Table 1). hobby servo, and can provide up to $4.3 \mathrm{~N} * \mathrm{~m}$ torque, more than any other hobby servo readily 
available and closest to the healthy human wrist maximum extension torque of $4.6 \pm 1.0 \mathrm{~N} * \mathrm{~m}$ [28] previously mentioned in the design criteria section. The two wrist motors are powered using a 7.5 V, 20 Amp power supply (967-CUS200LD7R5, TDK-Lambda Americas Inc., National City, CA).

Table 1: The wrist specifications fall within the established design criteria

\begin{tabular}{|c|c|}
\hline \multicolumn{2}{|c|}{ Wrist Specifications } \\
\hline $\begin{array}{c}\text { Degrees of } \\
\text { Freedom }\end{array}$ & $\begin{array}{c}\text { Pronation/Supination in series with Flexion/Extension or } \\
\text { Radial/Ulnar Deviation }\end{array}$ \\
\hline Length & $11.8 \mathrm{~cm}$ \\
\hline Weight & $360 \mathrm{~g}$ \\
\hline Range of & Pronation/Supination -180 Degrees \\
Motion & Radial/Ulnar Deviation or Flexion/Extension - Up to 175 Degrees \\
\hline Torque & $4.3 \mathrm{~N} * \mathrm{~m}$ (both motors) \\
\hline Cost & $<\$ 600$ \\
\hline
\end{tabular}

Assessment of Wrist Function:

To understand the usefulness of an active wrist, we modified the clothespin relocation task: a simple task that relies on wrist function [30]. The experiment was completed by naïve, non-amputee participants ( $\mathrm{n}=8$ participants) wearing a transradial bypass socket to simulate the experience of an amputee [29] (Figure 2). All participants gave written informed consent before taking part in experiments, in accordance with the University of Utah Institutional Review Board and the Department of Navy Human Research Protection Program. Participants were instructed to move a single clothespin from one location to another as fast as possible (See Prosthetic Control Paradigm section, below). If a participant dropped the clothespin, the trial was marked as a failure and discarded. Three variations of the task were completed (Figure 3A): a) moving the clothespin from a horizontal position to an adjacent horizontal position; b) moving the clothespin from a horizontal position to a vertical position (requiring wrist pronation/supination); c) reaching over a dowel to move the clothespin from a horizontal position to a horizontal position under the dowel (requiring wrist flexion/extension). 
(a)

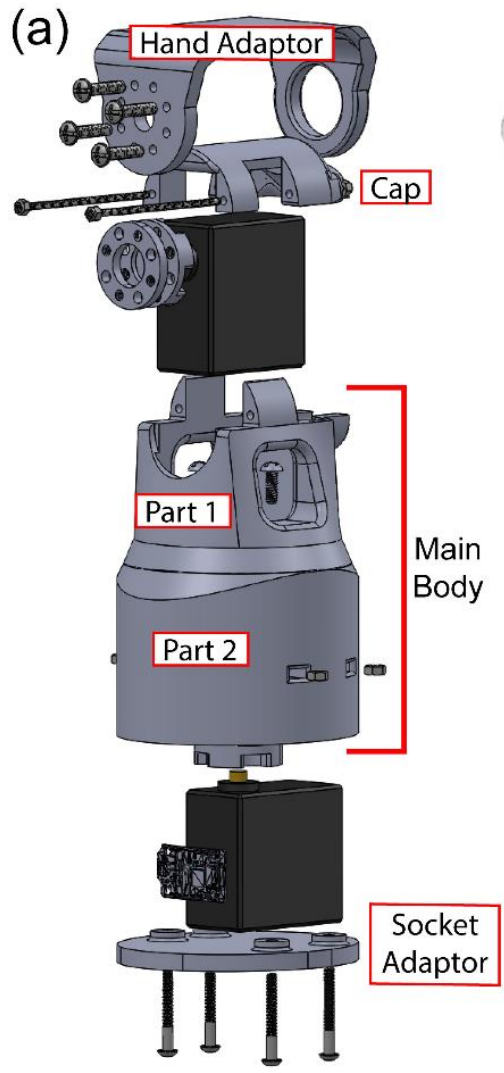

(b)

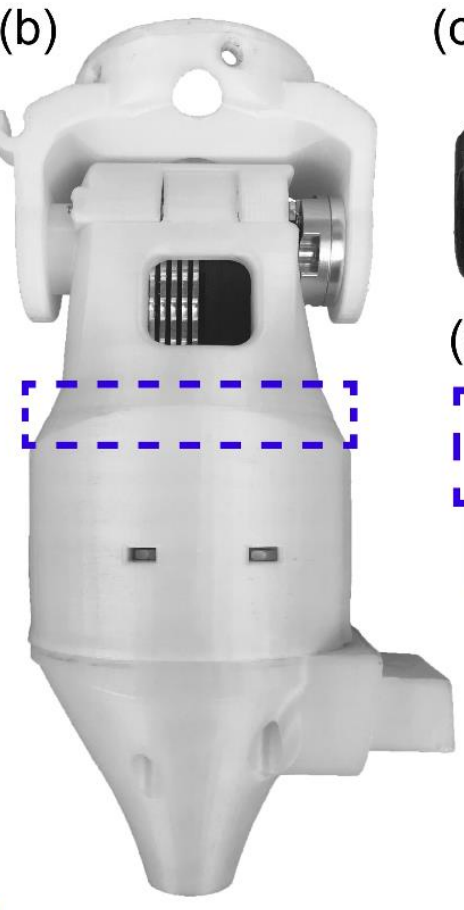

(c)
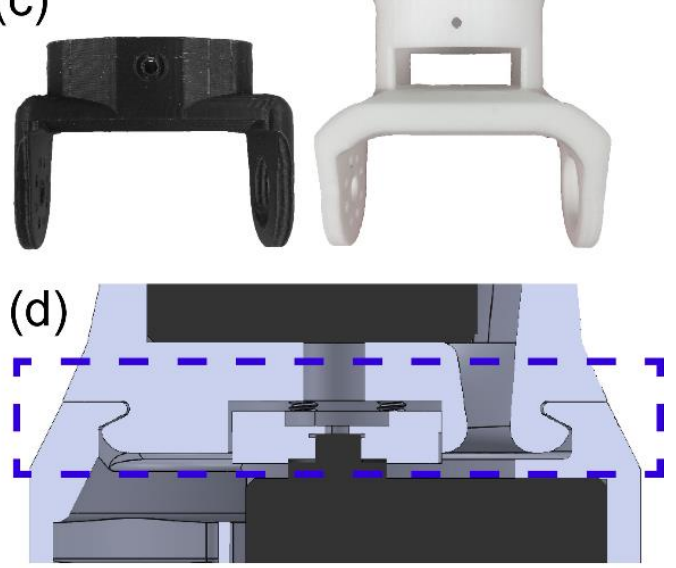

(e)

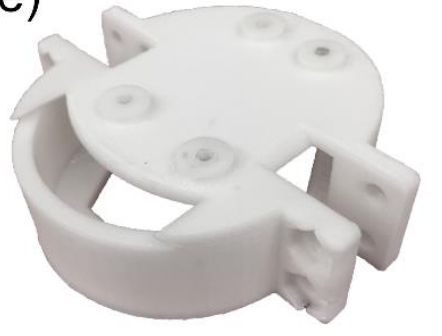

Figure 1. CAD design (a) and 3D-print (b) of the designed wrist. The hand adaptor (c) is easy to modify to fit to various prosthetic hands. The currently attached part (seen in b) is for the Ottobock quick disconnect. Attachments have also been made for the Ada [14] and Handi [16] hands. (d) The main body of the wrist contains subparts 1 and 2 which are 3D-printed with PLA and dissolvable PVA support material. These parts are interconnected via an interlocking rotational mechanism to preserve space. The proximal end can also be adapted to various sockets (e). Currently attached to the proximal end of the wrist in (b) is an attachment for the Bypass Socket [29].

Prosthetic Control Paradigm: A motion capture system (Leap Motion, San Francisco, CA) was mounted to the top of the wrist rotation mechanism of the bypass socket (Figure 2) to track the position of the participants hands and wrist. Motion capture provided precise joint angles sampled at $60 \mathrm{~Hz}$. Only a single degree of freedom (index finger flexion/extension) was provided to the hand to simplify the hand control and focus comparisons on wrist functionality. The index finger position of both hands was updated at $10 \mathrm{~Hz}$. Due to inaccessible underlying software restrictions, the control of the DEKA "LUKE” Arm wrist differed from the wrist developed herein. The DEKA “LUKE” Arm required wrist position data to be converted to a velocity control signal. For the developed wrist, the joint angles of the wrist were averaged over a 1 
second period to smooth the signal and used to control the wrist. For control conditions, both wrists were locked in a neutral position and was not actively updated with the motion tracking.

Experimental Conditions: Each participant completed the experiment under four conditions in a pseudorandomized counter-balanced crossover design, such that each participant served as their own control. The four experimental conditions were different hand/arm combinations, including: a) TASKA Hand with the 3D-printed wrist ( $\mathrm{T}+\mathrm{W}$, first experimental condition); b) TASKA Hand with the 3D-printed wrist in locked position (T-W, first control); c) DEKA "LUKE" Arm with its embedded wrist fully functional ( $\mathrm{L}+\mathrm{W}$, second experimental condition), and 4) DEKA "LUKE" Arm with its embedded wrist locked (L-W, second control). Our goal was to measure overall improvements associated with the wrist conditions $(+\mathrm{W})$ relative to the no-wrist conditions $(-\mathrm{W})$, such that the primary comparisons are $\mathrm{T}+\mathrm{W}$ vs. $\mathrm{T}-\mathrm{W}$, and $\mathrm{L}+\mathrm{W}$ vs. $\mathrm{L}-\mathrm{W}$.

The cross-over design was made up of 8 distinct experimental blocks performed one after another. Experimental blocks were pseudorandomized and counterbalanced to combat fatigue and motor learning effects. For each block, the participant performed the three tasks (horizontalhorizontal, horizontal-vertical, and over-under) four times each using a single hand-arm configuration. This resulted in a total of 8 trials per condition ( 2 blocks per condition, 4 trials per block). Participants were allotted up to 1 minute of practice before each block of trials. The median time of the 8 trials was reported as the participants final score.

Performance Metrics: We collected five performance metrics for each experimental condition: individual task completion time, workload across all tasks, and user preference across all tasks. The median time to completion (across the 8 trials) served as the completion time for each condition. After participants completed all tasks for a given experimental condition, workload was measured by having each participant complete the NASA Task Load Index survey [31]. The NASA Task workload score uses six workload-related factors to estimate an overall subjective workload score. At the end of the experiment, participants were asked to rank order the hand/wrist combination based on preference, such that a score of 1 is most preferable.

Statistical Analysis: All data were checked for normality using the Anderson-Darling and Lilliefors tests for normality. Outliers (more than 1.5 interquartile ranges above the upper quartile or below the lower quartile) were removed prior to statistical analyses. A one-way analysis of variance (ANOVA), or non-parametric equivalent (Kruskal-Wallis), was then 
191 statistical difference was found, subsequent pair-wise comparisons were performed using the

192 Dunn-Sidak correction for multiple comparisons.

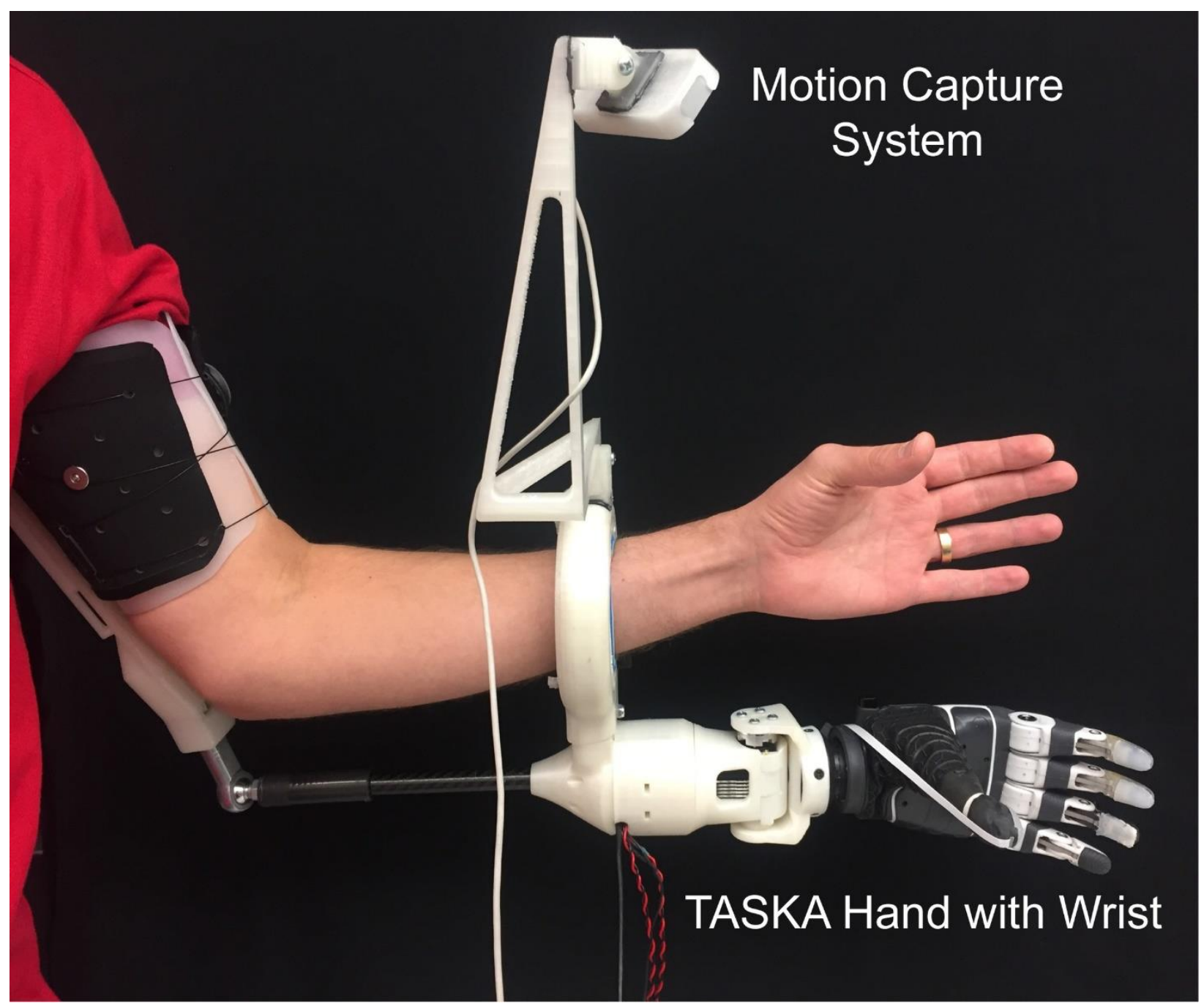

Figure 2. The intact participants wore a bypass socket [29] with a motion tracking system (Leap Motion) attached. Movement of the intact hand and wrist was tracked and translated into control of the 196 prosthetic hand and wrist.

\section{RESULTS}

\section{Wrist Performance and Assessment:}

199 There were no statistically reliable differences in time to completion for hands with functional wrists compared without functional wrists. However, providing a wrist reduced subjective 
201

202

203

204

205

206

207

208

209

210

211

212

213

214

215

216

217

218

219

220

221

222

223

224

225

workload for both the LUKE and TASKA hands, as measured on the NASA Task Load Index. Subjects also preferred the LUKE hand with a wrist, over a LUKE hand without a wrist.

Horizontal to Horizontal Task: The average time for the participants to complete the task was $4.67 \pm 0.40$ for the $\mathrm{L}-\mathrm{W}, 4.97 \pm 0.31$ for the $\mathrm{L}+\mathrm{W}$ case, $5.67 \pm 0.51$ for the $\mathrm{T}-\mathrm{W}$, and $5.57 \pm 0.56$ for the $\mathrm{T}+\mathrm{W}$ case (Figure 3B). The one-way ANOVA showed no significant differences among the four experimental conditions $(p=0.355)$.

Horizontal to Vertical Task: The average time for the participants to complete the task was 6.41 \pm 0.39 for the $\mathrm{L}-\mathrm{W}$ case, $7.05 \pm 0.63$ for the $\mathrm{L}+\mathrm{W}$ case, $8.94 \pm 0.76$ for the $\mathrm{T}-\mathrm{W}$ case, and $7.60 \pm$ 0.60 for the $\mathrm{T}+\mathrm{W}$ case (Figure $3 \mathrm{~B}$ ). The one-way ANOVA showed significant differences among the four experimental conditions $(p<0.05)$. We found no significant differences among the primary comparisons of interest (wrist + vs. wrist -); however, significant difference was found between T-W vs. L-W $(p=0.039)$.

Over to Under Task: The average time for the participants to complete the task was $10.12 \pm 0.59$ for the $\mathrm{L}-\mathrm{W}, 10.00 \pm 0.69$ for the $\mathrm{L}+\mathrm{W}$ case, $9.71 \pm 1.22$ for the $\mathrm{T}-\mathrm{W}$ case, and $10.88 \pm 0.99$ for the $\mathrm{T}+\mathrm{W}$ case (Figure $3 \mathrm{~B}$ ). The one-way ANOVA showed no significant differences among the four experimental conditions $(p=0.827)$.

NASA Task Load Index Scores: Functional wrists decreased subjects' subjective workload as measured by the NASA Task Load Index. The overall workload required to use the various hand/wrist combinations was $57.88 \pm 4.94$ for the $\mathrm{L}-\mathrm{W}$ case, $36.42 \pm 5.69$ for the $\mathrm{L}+\mathrm{W}$ case, $57.33 \pm 5.67$ for the $\mathrm{T}-\mathrm{W}$ case, and $35.71 \pm 5.16$ for the $\mathrm{T}+\mathrm{W}$ case (Figure $3 \mathrm{C}$ ). The one-way ANOVA showed significant differences among the four experimental conditions $(\mathrm{p}<0.05)$.

22 Significant differences were found in both primary comparisons of interest $(\mathrm{L}+\mathrm{W}$ vs. $\mathrm{L}-\mathrm{W}$ and $23 \mathrm{~T}+\mathrm{W}$ vs. $\mathrm{T}-\mathrm{W})$. The workload was significantly less for the $\mathrm{L}+\mathrm{W}$ vs. $\mathrm{L}-\mathrm{W}(p=0.0026)$ as well 24 as for the T+W vs. T-W $(p=0.0024)$. Secondary comparisons also showed a significant 25 difference in $\mathrm{T}+\mathrm{W}$ vs. $\mathrm{L}-\mathrm{W}(p=0.0018)$ and $\mathrm{T}-\mathrm{W}$ vs. $\mathrm{L}+\mathrm{W}(p=0.0033)$. 


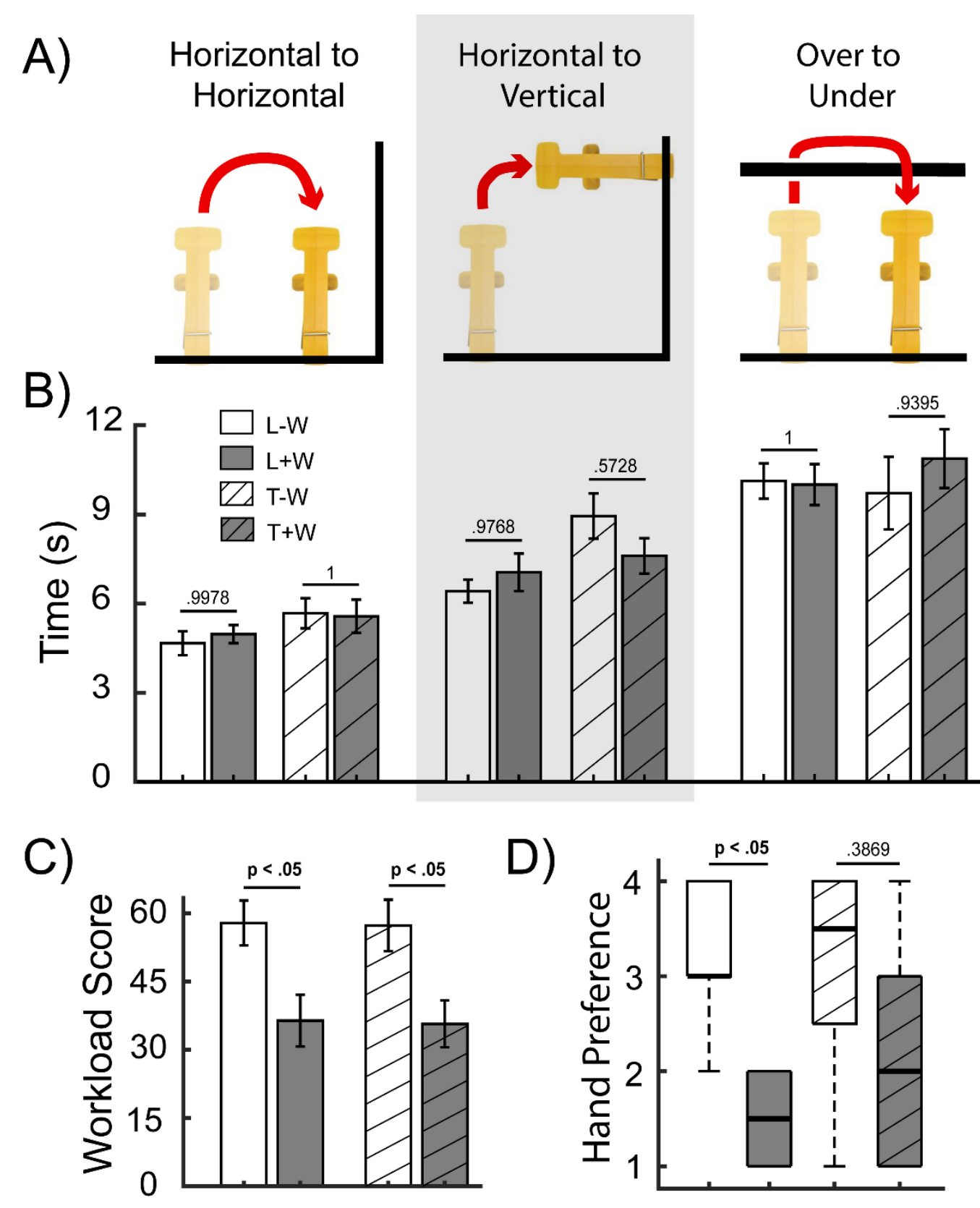

Figure 3. (A) The three variations of the clothes pin relocation task involved moving a clothespin from horizontal to horizontal position, horizontal to vertical position, and reaching over a horizontal bar to move the pin under the bar. (B) The implementation of a wrist did not significantly affect the time it took participants to complete tasks. (C) DEKA "LUKE" arm and TASKA workload scores were significantly reduced through the implementation of a wrist. (D) Arm preference as reported by participants for each condition. Bar plots show mean \pm S.E.M. Box plots show median (center line), interquartile range (box), and most extreme non-outlier values (whiskers). $N=8$ non-amputee participants. Significance differences were determined by a one-way ANOVA (or Kruskal-Wallis) followed by subsequent pairwise comparisons corrected for multiple comparisons. $p$ values are only shown for primary comparisons ( $T+W$ vs. T-W, and L+W vs. L-W). 
237 Subjective Hand Preference: The $\mathrm{L}+\mathrm{W}$ was selected first 4 times, second 4 times, third 0 times, 238 and fourth 0 times. The $\mathrm{T}+\mathrm{W}$ was selected first 3 times, second 2 times, third 2 times, and fourth

2391 time. The T-W was selected first 1 time, second 1 time, third 2 times, and fourth 4 times. The

240 L-W was selected first 0 times, second 1 time, third 4 times, and fourth 3 times. The average

241 choice of each hand combination was: 1.5 for $\mathrm{L}+\mathrm{W}, 2.125$ for $\mathrm{T}+\mathrm{W}, 3.125$ for $\mathrm{T}-\mathrm{W}$, and 3.25 for

242 L-W (Figure 3D). The Kruskal-Wallis showed statistical difference between the L+W vs. L-W ( $p$ $243=.0123)$. No statistical difference was found between the $\mathrm{T}+\mathrm{W}$ vs. $\mathrm{T}-\mathrm{W}(p=.3869)$. The lack of 244 statistical difference in the $\mathrm{T}+\mathrm{W}$ vs. $\mathrm{T}-\mathrm{W}$ case could be due to the $\mathrm{n}=8$ sample size. A secondary 245 comparison between the $\mathrm{L}+\mathrm{W}$ vs $\mathrm{T}-\mathrm{W}$ also showed statistical difference $(p=.0251)$.

246 Compensation: Figure 4 shows two individuals performing the horizontal to vertical and over to

247 under tasks. We observed that during the conditions without a wrist, the participants

248 compensated by rotating their shoulders and torso.

\section{DISCUSSION}

250 We developed a 3D-printable, lightweight, and adaptable prosthetic wrist, which met or 251 exceeded our design criteria (Table 1) and demonstrated that our wrist (as well as the DEKA 252 "LUKE" Arm's wrist) significantly reduced workload as measured by the NASA Task Load 253 Index in a modified clothespin relocation task. Participants preferred a wrist over no wrist for the $254 \mathrm{~L}+\mathrm{W}$ vs L-W comparison $(p=.0123)$. A larger sample size may be necessary to reveal 255 significance between the $\mathrm{T}+\mathrm{W}$ vs $\mathrm{T}-\mathrm{W}$ condition. Although not a primary comparison of interest, 256 for all 5 of the performance metrics, there were no significant differences between the $\mathrm{L}+\mathrm{W}$ and $257 \mathrm{~T}+\mathrm{W}$. This suggests that our wrist is not significantly worse than the commercially available DEKA "LUKE" Arm for these 5 performance metrics. 


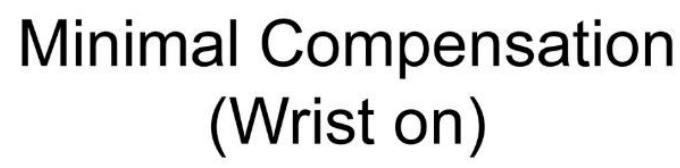

\section{Compensation (Wrist off)}
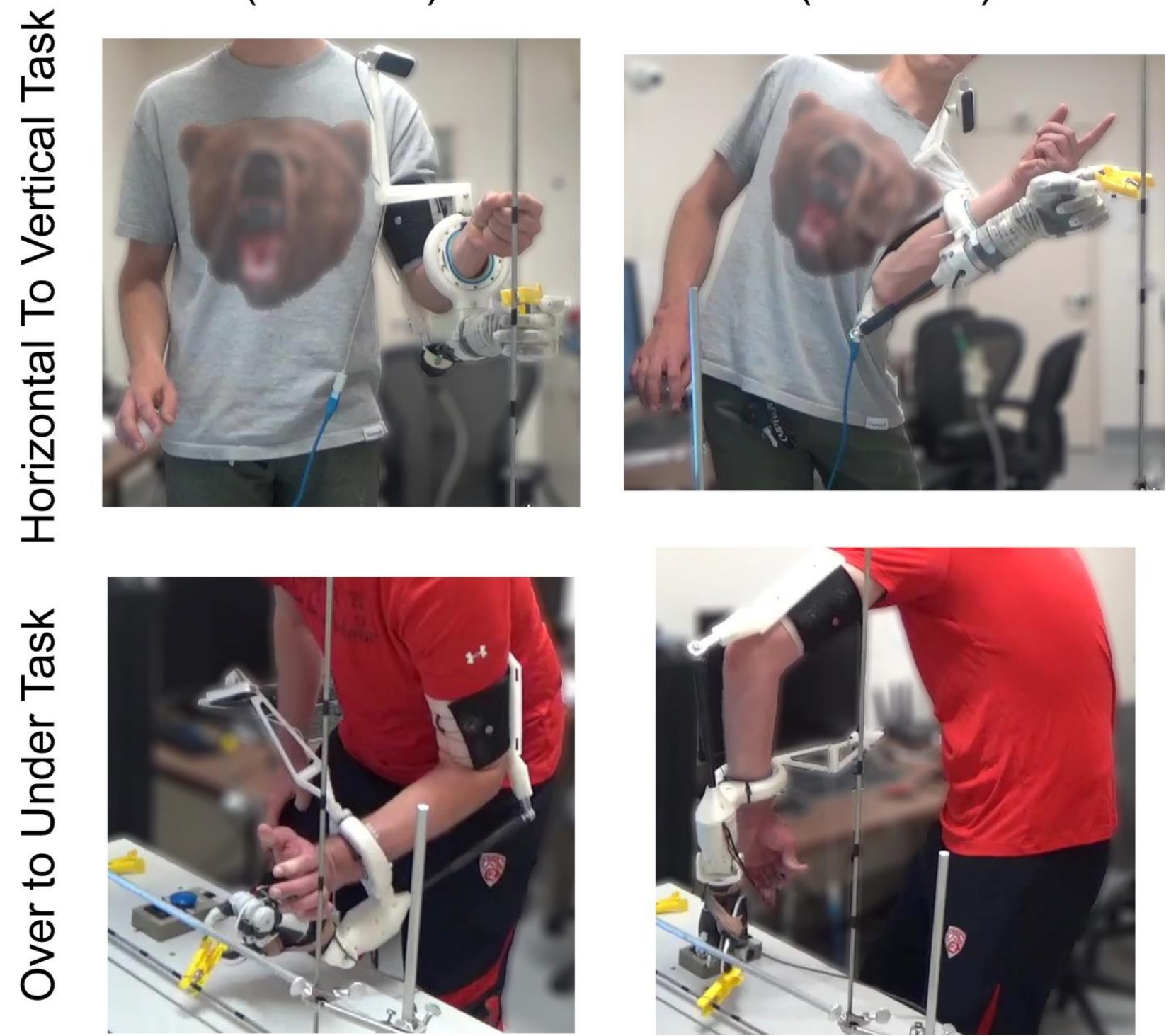

Figure 4. Participants relied on compensation to complete tasks that otherwise would require a wrist.

\section{Implementation of a Wrist Significantly Reduces Workload but Does Not Affect Speed}

Although having a wrist did not affect the time it takes for a participant to complete these particular tasks (Figure 3b), it did reduce the workload required for these tasks (Figure 3c).

265 Although we did not explicitly quantify compensatory movements, we visually observed that participants heavily used compensatory motions to complete the tasks when a wrist was not available (Figure 4). Anecdotally, and without explicit measurement, the addition of the wrist 
reduced compensatory movements (Figure 4). It is likely that the NASA Task Load index scores for workload were higher for the no-wrist cases due to the physical exertion required to use compensatory motions that the participant needed to perform to complete the tasks.

For timed tasks, statistical difference was only found in the time it took to complete the horizontal to vertical task with the T-W vs. L-W, which suggests that performance of the two hands differ. This could be due to differences in weight, grip strength, grip material or hand properties. For example, the DEKA “LUKE” Arm flexion and extension are coupled with radial and ulnar deviation. With the addition of the wrist, differences between the DEKA "LUKE" Arm vs. the TASKA hand were no longer present, suggesting that the wrist may preferentially benefit less functional hands. Comparing the $\mathrm{T}+\mathrm{W}$ and $\mathrm{L}+\mathrm{W}$ conditions was not our primary goal, but we observed no statistical differences among these conditions.

After the experiment, participants were asked to rank their preference of the prosthetic in order from 1-4 (Figure 3d). Collapsing across the two prosthetics, the experimental conditions without a functional wrist required a greater workload $(p<0.05$, paired t-test) and were preferred less ( $p<0.05$, Wilcoxon rank-sum test). Together, these results suggest that, despite there being no significant improvement in the time it took the participants to complete tasks, the addition of a functional wrist is preferable because it reduces the subjective workload needed to complete tasks.

\section{Other Developed Wrists Have Limitations in Length or Strength}

Other 1-, 2-, and 3-degree-of-freedom active prosthetic wrists have been developed.

However, few of these wrists can be 3D-printed and/or provide as much torque [22,23] (Table 2). One 2-degree-of-freedom wrist uses a differential system to conserve space. At just $4.8 \mathrm{~cm}$, the wrist is very short; however, it provides just $0.073 \mathrm{~N}^{*} \mathrm{~m}$ of torque for wrist flexion [32], which would not lift most prosthetic hands. The wrist discussed in this paper, though longer, uses motors that are much more practical and that can provide roughly 60 times more torque (4.3 $\mathrm{N}^{*} \mathrm{~m}$ ), which is much more realistic for of daily living.

Another wrist, developed by Dange [33], was capable of three degrees of freedom. This wrist is more than double the length of our wrist, making the wrist the length of the radius bone itself. The Dange wrist uses servo motors that provide the same torque and are the same dimensions as 
297 the ones used by our wrist. However, our wrist is far more compact and realistic as a prosthetic 298 device, although limited to only 2 degrees of freedom.

Table 2: Adapted from Bajaj Et. Al [23] to include only active wrists with more than 1 degree-of-freedom with developed wrist

\begin{tabular}{|l|c|c|c|c|c|c|c|}
\hline $\begin{array}{c}\text { Product Name/Lead } \\
\text { Author }\end{array}$ & DOF & 3D Printed & Commercial & $\begin{array}{c}\text { Diameter } \\
(\mathbf{c m})\end{array}$ & $\begin{array}{c}\text { Length } \\
(\mathbf{c m})\end{array}$ & $\begin{array}{c}\text { Weight } \\
(\mathbf{g})\end{array}$ & $\begin{array}{c}\text { Stall Torque } \\
(\mathbf{N * m m})\end{array}$ \\
\hline Olsen, N. R. & 2 & $Y$ & $N$ & 7.4 & 11.8 & 330 & 4300 \\
\hline Abd Razak, N. A. [34] & 2 & $\mathrm{~N}$ & $\mathrm{~N}$ & -- & -- & 500 & 13000 \\
\hline Controzzi, M. [35] & 2 & $\mathrm{~N}$ & $\mathrm{~N}$ & -- & -- & 240 & -- \\
\hline Kyberd, P. J. [32] & 2 & $\mathrm{~N}$ & $\mathrm{~N}$ & 9.6 & 5.0 & 200 & 73 \\
\hline Roose, C. [36] & 2 & $\mathrm{Y}$ & $\mathrm{N}$ & 5.3 & -- & 95 & $321(\mathrm{flexion)}$ \\
\hline RIC Arm [37] & 2 & $\mathrm{~N}$ & $\mathrm{~N}$ & -- & -- & $170 / 155$ & $900 / 1000$ \\
\hline $\begin{array}{l}\text { RSL Stepper bebionic } \\
\text { Wrist [38] }\end{array}$ & 2 & $\mathrm{~N}$ & $\mathrm{Y}$ & 5.0 & 7.5 & -- & -- \\
\hline Mahmoud, R. [39] & 3 & $\mathrm{Y}$ & $\mathrm{N}$ & - & - & - & $930 / 210 / 210$ \\
\hline Dange, S.V. (24) & 3 & $\mathrm{Y}$ & $\mathrm{N}$ & 10.16 & 26.67 & -- & 4300 \\
\hline
\end{tabular}

Due to the relatively limited availability of amputees, the experiment was performed on only intact participants using a bypass socket [29]. Paskett et al. showed that the bypass socket can closely simulate the experience of an amputee; however, future studies should broaden the testing of the wrist to include amputees.

One limitation of the servo motors is that extensive use at maximum load produces heat capable of softening the PLA. Future work would perform tests to quantify the extent of this overheating and could include efforts to reduce overheating by replacing servos with brushless motors (which draw less current and therefore create less heat), using a more heat resistant 3D-

311 printing material, or adding an explicit heat sink and cooling vents. a single-degree-of-freedom prosthetic hand with a wrist may be comparable to a dexterous hand

314 without a wrist (10). However, this study involved the use of an intact person with a brace on 
315

316

317

318

319

320

321

322

323

324

325

326

327

328

329

330

331

332

333

334

335

336

337

338

339

340

341

342

their arm limiting certain movements. Our wrist could extend this study and allow experimentation with a variety of prosthetic devices instead of an intact hand. The adaptable aspect of our wrist also enables it to be used to study optimal control algorithms for prosthetic wrists.

\section{CONCLUSION}

Our novel 3D printed wrist can be used with many terminal devices and sockets to reduce task load. The wrist can also be used in research to show how prosthetic devices can be improved with the implementation of a functional wrist. Ultimately the wrist may aid in the development of more biomimetic prosthetic devices and improve the quality of life for amputees.

Acknowledgments: We thank TASKA Prosthetics for the use of the TASKA hand in this study. We likewise thank DEKA for the use of the "LUKE" arm. We would also like to acknowledge the University of Utah Center for Medical Innovation for assisting us with the 3D printing of the wrist.

Funding: This work was sponsored by the Hand Proprioception and Touch Interfaces (HAPTIX) program administered by the Biological Technologies Office (BTO) of the Defense Advanced Research Projects Agency (DARPA), through the Space and Naval Warfare Systems Center (contract no. N66001-15-C-4017). Additional sponsorship was provided by the NSF through grant no. NSF ECCS-1533649 and NSF GRFP award no. 1747505.

\section{Author contributions:}

N.R.O. designed and built adaptable wrist, developed software for the wrist, designed the experiment, ran the experiments, 3D printed components for the experiment, performed statistical analysis, and drafted the manuscript.

J.A.G. provided design oversight, developed software to control the prosthetics, designed the experiment, provided guidance on statistical methods, and drafted the manuscript.

M.R.B. designed the experiment, provided guidance on statistical methods, and drafted the manuscript. 
343 M.D.P provided wrist design oversight, developed the bypass socket used in the experiment, and 344 provided guidance on statistical methods.

345 D.T.K. provided wrist design oversight, and designed software to control the prosthetics.

346 T.N.T. developed software to control the prosthetics.

347 C.C.D. provided clinical support, oversights, and relevance throughout.

348 G.A.C. oversaw and led the development of all methods, experiments, and protocols and assisted 349 with experiments and drafting of the manuscript.

350 All authors contributed to the revision of the manuscript. The views expressed herein are the 351 authors' and do not necessarily reflect the views of their employers or funding sources.

Competing interests: D.T.K. is now an employee of Blackrock Microsystems.

Data and materials availability: Deidentified data will be made available upon request. Data and materials requests should be sent to G.A.C. (greg.clark@utah.edu). Distribution of 356 identifiable data for human subjects will require approval of the University of Utah Institutional 357 Review Board.

\section{REFERENCES}

1. Ziegler-Graham K, MacKenzie EJ, Ephraim PL, Travison TG, Brookmeyer R. Estimating the Prevalence of Limb Loss in the United States: 2005 to 2050. Arch Phys Med Rehabil. 2008;89: 422429. doi:10.1016/j.apmr.2007.11.005

2. Bhuvaneswar CG, Epstein LA, Stern TA. Reactions to Amputation: Recognition and Treatment. Prim Care Companion J Clin Psychiatry. 2007;9: 303-308.

3. Desmond DM, MacLachlan M. Affective distress and amputation-related pain among older men with long-term, traumatic limb amputations. J Pain Symptom Manage. 2006;31: 362-368. doi:10.1016/j.jpainsymman.2005.08.014

4. Biddiss E, Chau T. Upper-Limb Prosthetics: Critical Factors in Device Abandonment. Am J Phys Med Rehabil. 2007;86: 977-987. doi:10.1097/PHM.0b013e3181587f6c

5. Atkins DJ, Heard DCY, Donovan WH. Epidemiologic Overview of Individuals with Upper-Limb Loss and Their Reported Research Priorities. JPO J Prosthet Orthot. 1996;8: 2. prosthesis users during common tasks. Clin Biomech. 2008;23: 1128-1135. 
7. Bertels T, Schmalz T, Ludwigs E. Objectifying the Functional Advantages of Prosthetic Wrist Flexion: JPO J Prosthet Orthot. 2009;21: 74-78. doi:10.1097/JPO.0b013e3181a10f46

8. Safaee-Rad R, Shwedyk E, Quanbury AO, Cooper JE. Normal functional range of motion of upper limb joints during performance of three feeding activities. Arch Phys Med Rehabil. 1990;71: 505509.

9. Mell AG, Childress BL, Hughes RE. The Effect of Wearing a Wrist Splint on Shoulder Kinematics During Object Manipulation. Arch Phys Med Rehabil. 2005;86: 1661-1664. doi:10.1016/j.apmr.2005.02.008

10. Østlie K, Franklin RJ, Skjeldal OH, Skrondal A, Magnus P. Musculoskeletal Pain and Overuse Syndromes in Adult Acquired Major Upper-Limb Amputees. Arch Phys Med Rehabil. 2011;92: 1967-1973.e1. doi:10.1016/j.apmr.2011.06.026

11. Slade P, Akhtar A, Nguyen M, Bretl T. Tact: Design and performance of an open-source, affordable, myoelectric prosthetic hand. 2015 IEEE International Conference on Robotics and Automation (ICRA). Seattle, WA, USA: IEEE; 2015. pp. 6451-6456. doi:10.1109/ICRA.2015.7140105

12. ten Kate J, Smit G, Breedveld P. 3D-printed upper limb prostheses: a review. Disabil Rehabil Assist Technol. 2017;12: 300-314. doi:10.1080/17483107.2016.1253117

13. Zuniga J, Katsavelis D, Peck J, Stollberg J, Petrykowski M, Carson A, et al. Cyborg beast: a low-cost 3d-printed prosthetic hand for children with upper-limb differences. BMC Res Notes. 2015;8: 10. doi:10.1186/s13104-015-0971-9

14. Ada V1.1 Assembly Instructions. In: Open Bionics [Internet]. [cited 9 Sep 2019]. Available: https://openbionicslabs.com/obtutorials/ada-v1-assembly

15. Brunel Hand 2.0. In: Open Bionics [Internet]. [cited 9 Sep 2019]. Available: https://openbionicslabs.com/shop/brunel-hand

16. Brenneis DJA, Dawson MR, Pilarski PM. DEVELOPMENT OF THE HANDI HAND : AN INEXPENSIVE, MULTI-ARTICULATING , SENSORIZED HAND FOR MACHINE LEARNING RESEARCH IN MYOELECTRIC CONTROL. 2017.

17. Montagnani F, Controzzi M, Cipriani C. Is it Finger or Wrist Dexterity That is Missing in Current Hand Prostheses? IEEE Trans Neural Syst Rehabil Eng. 2015;23: 600-609.

doi:10.1109/TNSRE.2015.2398112

18. Michelangelo hand prosthesis. [cited 11 Sep 2019]. Available: https://www.ottobock.com.au/prosthetics/upper-limb/solution-overview/axon-bus-prostheticsystem-with-michelangelo-hand/

19. i-Limb Ultra. [cited 9 Sep 2019]. Available: https://www.ossur.com/prostheticsolutions/products/touch-solutions/i-limb-ultra\#accessories

20. Advanced Prosthetic Hand | Taska Prosthetics. [cited 9 Sep 2019]. Available: http://www.taskaprosthetics.com/the-taska/features/ 
21. bebionic hand. [cited 9 Sep 2019]. Available: https://www.ottobockus.com/prosthetics/upperlimb-prosthetics/solution-overview/bebionic-hand/

22. Bajaj NM, Spiers AJ, Dollar AM. State of the Art in Artificial Wrists: A Review of Prosthetic and Robotic Wrist Design. IEEE Trans Robot. 2019;35: 261-277. doi:10.1109/TRO.2018.2865890

23. Bajaj NM, Spiers AJ, Dollar AM. State of the art in prosthetic wrists: Commercial and research devices. 2015 IEEE International Conference on Rehabilitation Robotics (ICORR). Singapore, Singapore: IEEE; 2015. pp. 331-338. doi:10.1109/ICORR.2015.7281221

24. LUKE Arm Detail Page - Mobius Bionics. [cited 9 Sep 2019]. Available:

http://www.mobiusbionics.com/luke-arm/

25. Johannes MS, Bigelow JD, Burck JM, Harshbarger SD, Kozlowski MV, Doren TW van. An Overview of the Developmental Process for the Modular Prosthetic Limb. 2011.

26. Mall G, Hubig M, Büttner A, Kuznik J, Penning R, Graw M. Sex determination and estimation of stature from the long bones of the arm. Forensic Sci Int. 2001;117: 23-30. doi:10.1016/S03790738(00)00445-X

27. Biddiss $E$, Beaton $D$, Chau T. Consumer design priorities for upper limb prosthetics. Disabil Rehabil Assist Technol. 2007;2: 346-357. doi:10.1080/17483100701714733

28. Yoshii $Y$, Yuine $H$, Kazuki $O$, Tung $W$, Ishii T. Measurement of wrist flexion and extension torques in different forearm positions. Biomed Eng OnLine. 2015;14. doi:10.1186/s12938-015-0110-9

29. Paskett MD, Olsen NR, George JA, Kluger DT, Brinton MR, Davis TS, et al. A Modular Transradial Bypass Socket for Surface Myoelectric Prosthetic Control in Non-Amputees. ArXiv190903153 Cs QBio. 2019 [cited 11 Sep 2019]. Available: http://arxiv.org/abs/1909.03153

30. Kyberd P, Hussaini A, Maillet G. Characterisation of the Clothespin Relocation Test as a functional assessment tool. J Rehabil Assist Technol Eng. 2018;5: 2055668317750810. doi:10.1177/2055668317750810

31. Hart SG, Staveland LE. Development of NASA-TLX (Task Load Index): Results of Empirical and Theoretical Research. In: Hancock PA, Meshkati N, editors. Advances in Psychology. North-Holland; 1988. pp. 139-183. doi:10.1016/S0166-4115(08)62386-9

32. Kyberd PJ, Lemaire ED, Scheme E, MacPhail C, Goudreau L, Bush G, et al. Two-degree-of-freedom powered prosthetic wrist. J Rehabil Res Dev. 2011;48: 609. doi:10.1682/JRRD.2010.07.0137

33. Dange SV. Design of a working model of an upper limb prosthesis: wrist mechanism. 2017. doi:10.7282/T30G3P8X

34. Abd Razak NA, Abu Osman NA, Gholizadeh H, Ali S. Development and performance of a new prosthesis system using ultrasonic sensor for wrist movements: a preliminary study. Biomed Eng OnLine. 2014;13: 49. doi:10.1186/1475-925X-13-49 
35. Controzzi M, Cipriani C, Jehenne B, Donati M, Carrozza MC. Bio-inspired mechanical design of a tendon-driven dexterous prosthetic hand. 2010 Annual International Conference of the IEEE Engineering in Medicine and Biology. 2010. pp. 499-502. doi:10.1109/IEMBS.2010.5627148

36. Roose C. Two-degree-of-freedom pneumatically powered wrist prosthesis. 2014 [cited 7 Sep 2019]. Available: https://repository.tudelft.nl/islandora/object/uuid\%3A60cc243c-20d0-4ead80d3-0bc29878e679

37. Lenzi T, Lipsey J, Sensinger JW. The RIC Arm-A Small Anthropomorphic Transhumeral Prosthesis. IEEEASME Trans Mechatron. 2016;21: 2660-2671. doi:10.1109/TMECH.2016.2596104

38. Varley E. An Artificial Joint. WO/2012/098347, 2012. Available: https://patentscope. wipo.int/search/en/detail.jsf?docld=WO2012098347

39. Mahmoud R, Ueno A, Tatsumi S. Dexterous mechanism design for an anthropomorphic artificial hand: Osaka City University Hand I. 2010 10th IEEE-RAS International Conference on Humanoid Robots. 2010. pp. 180-185. doi:10.1109/ICHR.2010.5686843 